\title{
Estudio psicométrico del Cuestionario de Detección del Trastorno de Conducta (CDTC)
}

\author{
Ferran Padrós-Blázquez ${ }^{1}$, Arly Danahé Olavarrieta², Maria Patricia Martinez-Medina ${ }^{3}$ y \\ Fabiola González-Betanzos ${ }^{2}$
}

\begin{abstract}
RESUMEN
En México, existe un elevado índice de violencia y este se ha ido incrementando a lo largo de los años. Un alto porcentaje de estos individuos cumplen con los criterios del Trastorno Antisocial de la Personalidad en la edad adulta o del Trastorno de Conducta (TC) en la niñez o adolescencia. El comportamiento de estos sujetos es agresivo y existe una violación de las reglas y de los derechos de los demás. Ya que el TC conlleva consecuencias graves y puede progresar a un trastorno antisocial, es conveniente atender a los niños y adolescentes con la mayor brevedad posible. Por ello, se necesita disponer de un instrumento para el diagnóstico del TC en México. Con el objetivo de contar con una herramienta confiable se analizaron las propiedades del cuestionario basado en los criterios del DSM-IV para el diagnóstico del trastorno disocial (nombre del trastorno en el DSM-IV-TR, actualmente (TC) con una muestra de 305 estudiantes de escuela de secundaria, 155 de género femenino y 150 del masculino de la ciudad de Morelia, Michoacán (México). Se concluyó que la mejor solución fue de 1 factor en cuanto a su estructura interna y un valor del alfa de Cronbach de .87. Los 14 reactivos resultaron adecuados. Se realizaron correlaciones de Pearson con otras medidas y se obtuvieron correlaciones positivas y moderadas con la Escala de Impulsividad de Plutchik, con el Inventario de Depresión de Beck y con la escala CRAFFT de Knight (evalúa riesgo de abuso de sustancias) y se observó la ausencia de correlación con el Inventario de Ansiedad estado-rasgo. Se concluye que este instrumento posee adecuadas propiedades psicométricas para ser administrado en la ciudad de Morelia (México).
\end{abstract}

Palabras clave: trastorno de conducta, trastorno disocial, agresión, violencia, validación.

\section{Psychometric Study of the Detection Questionnaire for Conduct Disorder}

\begin{abstract}
In Mexico an elevated rate of violence exists and it has been increasing over the years. A high percentage of these violent individuals fulfill the criteria of Antisocial Personality Disorder in their adult life or Conduct Disorder (CD) in their childhood or adolescence. The behavior of these individuals is aggressive and there is a violation of the rules and the rights of others. Since CD carries serious consequences and can progress to an antisocial disorder, it is advisable to treat children and adolescents as soon as possible. Therefore, it is necessary to have an instrument for the diagnosis of CD in Mexico. In order to have a reliable tool, the DSM-IV questionnaire was analyzed for the diagnosis of the conduct disorder (Previously dissocial disorder in DSM-IV-TR) with a sample of 305 high school students, 155 female and 150 male students from the city of Morelia, Michoacán (Mexico). It was concluded that the best solution was 1 factor in terms of its internal structure and a Cronbach alpha value of .87. All 14 items were suitable, Pearson's correlations were made with other measures, obtaining positive and moderate correlations with the Plutchik Impulsivity Scale, the Beck Depression Inventory and the
\end{abstract}

1, 2 y 4 Universidad Michoacana de San Nicolás de Hidalgo, México; fpadros@umich.mx

${ }^{3}$ Centro Michoacano de Salud Mental, Mexico. 
Knight CRAFFT scale (Assess risk of substance abuse), and there was no correlation with the State-Trait Anxiety Inventory. Results support that this instrument possesses adequate psychometric properties to be administrated on the city of Morelia, Michoacán (Mexico).

Keywords: conduct disorder, dissocial disorder, aggression, violence, validation.

Los comportamientos agresivos y la desobediencia en el niño y el adolescente son causantes de preocupaciones en el ámbito familiar. Muchos padres ocasionalmente deben afrontar conductas de sus hijos que consisten en peleas, mentiras, discusiones o incluso destrucciones materiales (PadrósBlázquez et al. 2015). Sin embargo, cuando estas conductas son repetitivas y persistentes en extremo, llegan a afectar significativamente actividades académicas, familiares y sociales y pueden convertirse en un trastorno de la personalidad (Padrós-Blázquez et al., 2015). En los individuos con trastorno de conducta (TC) según criterios del DSM-5 (American Psychiatric Association, 2013) se observan comportamientos diferentes a la conducta normal (la cual ha sido establecida por la sociedad), que se consideran perjudiciales para esta y exigen una intervención (Staff, Machleidt, \& Bauer 2004).

\section{Características del trastorno de conducta}

En el DSM-5 se define el Trastorno de Conducta (TC), denominado Trastorno Disocial en su versión anterior DSM-IV y IV-TR (American Psychiatric Association, 1994; 2000) como "un patrón repetitivo y persistente de comportamiento en el que no se respetan los derechos básicos de otros, las normas o reglas sociales propias de la edad" (American Psychiatric Association, 2013, p. 469). Se distinguen cuatro tipos de comportamientos a los que hace referencia el criterio A: 1) Agresión a personas y animales; 2) Destrucción de la propiedad; 3) Engaño o robo; 4) Incumplimiento grave de las normas. Sin embargo, se exige la presencia de tres (o más) de los 15 criterios (distribuidos en los cuatro tipos), independientemente de la categoría de comportamiento, durante los últimos 12 meses y por lo menos de un criterio durante los últimos 6 meses. Por otro lado, el criterio B se refiere al deterioro que la alteración provoca a la persona, el cual debe ser clínicamente significativo en la actividad social, académica o laboral. Finalmente, el criterio $\mathrm{C}$ alude a la exclusión del cumplimiento de los criterios de trastorno antisocial de la personalidad en el caso de que el evaluado tenga 18 o más años (American Psychiatric Association, 2013). De tal modo que los criterios no se han modificado respecto de los del DSM-IV y IV-TR (American Psychiatric Association, 1994; 2000) a pesar del cambio de su nombre.

Por otro lado, debido a los avances en la investigación (Frick \& Nigg, 2012; Moffitt et al., 2008) en el DSM-5, aparte de los especificadores con respecto a la edad de inicio y de la gravedad, se incluye un nuevo especificador para el trastorno etiquetado como con emociones prosociales limitadas, que se aplica a aquellos sujetos que muestran una capacidad limitada de empatía, remordimiento, culpa, además de exhibir despreocupación por su rendimiento y mostrar un afecto superficial o deficiente (American Psychiatric Association, 2013). Dicho especificador se asocia al constructo de callo emocional, que ha sido descrito como una dimensión afectiva (se caracteriza por rasgos como ausencia de empatía, falta de remordimientos, falta de preocupación por el 
mal desempeño personal en el trabajo o la escuela y una expresión deficiente del afecto o afecto empalagoso). Estos rasgos parecen ser relativamente estables durante la infancia y la adolescencia y se asocian a un comportamiento más agresivo y persistente (Frick \& White, 2008).

Según el DSM-5 (American Psychiatric Association, 2013), el TC es un trastorno muy frecuente cuya prevalencia de un año (porcentaje de individuos que cumplen con los criterios diagnósticos en un período de 12 meses) se estima entre el 2 y el $10 \%$ (con una media del $4 \%$ ) y cuyos datos son bastante consistentes en los estudios de diferentes países. En los varones la prevalencia es mayor. En un estudio realizado en Colombia se observó el TC en un $8.8 \%$ de la población masculina y en un $2.7 \%$ en la población femenina (PosadaVilla, Aguilar-Gaxiola, Magaña, \& Gómez, 2004). En México, según datos de la Encuesta Nacional de Epidemiologia Psiquiátrica (Medina-Mora et al., 2003), la prevalencia del TC en algún momento de la vida se estimó hasta en un 10.3 $\%$ para los varones y de un $2.3 \%$ para el sexo femenino. En un estudio transnacional reciente con jóvenes estudiantes de entre 18 y 22 años se estimó una prevalencia del TC de 2.8 \% (Auerbach et al., 2016).

El TC suele iniciarse sobre los 10 años de edad de una persona y muestra el mayor predomino sobre los 14; además se asocia con los trastornos por uso de sustancias, así como con abandono escolar, conductas sexuales de riesgo, arrestos, entre otros (Greenfield, Sittner, Forbes, Walls, \& Whitbeck, 2017). El trastorno por déficit de atención con hiperactividad (TDAH) puede contemplarse como un factor de riesgo para el TC y este posteriormente como una vía para el trastorno por uso de sustancias, especialmente el alcohol (Padrós-Blázquez, Zamora-Cervantes, Salinas-García, \& Martínez-Medina, 2017) debido a que se ha descrito una elevada comorbilidad entre el TDAH y el TC (Ulloa, Sánchez, Sauceda, \& Ortiz, 2006). Beauchaine, Hinshaw y Pang (2010) mencionan que la mayoría de los adolescentes con un diagnóstico temprano de TC también cumplen con los criterios necesarios para el trastorno por déficit de atención e hiperactividad, lo que indica que el rasgo de impulsividad es común en ambos trastornos. Anney et al. (2008) señalan que una elevada impulsividad es característica de los TC y del abuso de sustancias, entre otros. Además, se ha reportado un mayor riesgo de sufrir otros trastornos como los del estado de ánimo, los de ansiedad y los somatoformes (Wiener \& Dulcan, 2006).

\section{Evaluación del trastorno de conducta}

Para evaluar el TC se requiere de información de diferentes participantes: el propio niño o adolescente; los padres o tutores; los profesores, e incluso a veces se cuenta con información derivada de la corte, ya que algunos inician procesos judiciales acusados de cometer delitos. Muchas veces es necesario realizar entrevistas exhaustivas y utilizar diferentes instrumentos de evaluación para llegar al diagnóstico. Existen instrumentos dirigidos a los padres, como el Eyberg Child Behavior Inventory (Eyberg, 1992), que se utiliza para medir los problemas conductuales. Este instrumento arroja resultados sobre la frecuencia en la que los niños manifiestan 36 conductas diferentes, asimismo evalúa si los padres valoran ciertos comportamientos como problemas. Dicho instrumento se puede aplicar además como un pre-test y post-test para evaluar la efectividad de tratamientos (McNeil \& Hembree-Kigin, 2010). También se han desarrollado cuestionarios dirigidos al propio niño o adolescente, que se centran en conducta delictiva; algunos muy largos, como 
por ejemplo el Questionnaire for the International Study on Self-Report Delinquency desarrollado en Holanda por Marshall et al. (1991, como se citó en Redondo \& Sánchez-Meca, 2003), el cual consta de un total de 574 ítems (Redondo, Garrido, Pérez, \& Barberet, 1997) además de otros instrumentos que evalúan la presencia de conducta criminal y delictiva; puede consultarse una revisión en el trabajo de Thornberry y Krohn (2000). Pero se puede también evaluar el TC o el trastorno disocial a partir de una lista de sintomas (checklist) basada en los que aparecen en los manuales diagnósticos clasificatorios como el DSM o la CIE. Pineda, Puerta, Arango, Calad y Villa (2000) construyeron el cuestionario breve para el diagnóstico del trastorno disocial de la conducta a partir de los sintomas que aparecen en el criterio A del DSM-IV (y que coinciden fundamentalmente con los del DSM-5) y lo aplicaron a adolescentes de 12 a 16 años en la ciudad de Medellín, Colombia. El mismo consta de 14 preguntas que se responden mediante una escala de Likert de 4 posiciones Nunca, Algunas veces, Frecuentemente y Siempre.

El cuestionario obtuvo una consistencia interna aceptable $(\alpha=.86)$. Se realizó un análisis factorial exploratorio y se obtuvieron 3 factores. En el primer factor se agrupan los ítems que describen "violación de normas" el cual explica el $32.9 \%$ de la varianza. El segundo factor está conformado por los items que describen "violencia" y explica el $10.9 \%$. Por último, el tercer factor etiquetado como "crueldad" explica un $10.1 \%$ de la varianza. Lo anterior, hace del cuestionario un instrumento de gran utilidad para la detección de casos de riesgo de padecer el TC a modo de cribaje. Debe destacarse que en la valoración de las puntuaciones totales se observa que hasta un $45 \%$ de la muestra evaluada en el estudio de Pineda et al. (2000) obtuvo una puntuación igual o superior a 4 (el punto de corte que sugieren los autores de riesgo de $\mathrm{TC})$.

Tomando en consideración que el Cuestionario para el Diagnóstico del Trastorno de Conducta (CDTC) de Pineda et al. (2000) ha mostrado aceptables propiedades psicométricas, que es un instrumento breve y que actualmente en México no se dispone de ningún instrumento para detectar el TC, se propuso como principal objetivo de la presente investigación estudiar algunas características psicométricas del CDTC en la población de Morelia, Michoacán (México). Los objetivos específicos fueron: 1) Estudiar la estructura interna del instrumento; 2) Estudiar la confiabilidad de la escala y la bondad de los reactivos; 3) Estudiar la relación con otras variables (impulsividad, depresión, ansiedad y abuso de sustancias) y ofrecer datos descriptivos de la muestra.

\section{MÉTODO}

\section{Participantes}

Los participantes fueron 305 adolescentes de entre 12 y 15 años, estudiantes de escuela secundaria. Se realizó un muestreo por conveniencia. La edad media resultó de $13.32(D E=0.97)$, donde $155(50.8 \%)$ eran del sexo femenino y 150 (49.2\%), del masculino.

\section{Instrumentos}

Se aplicaron 5 instrumentos que se describen a continuación:

El Cuestionario basado en los criterios del DSM-IV (American Psychiatric Association, 1994) para el diagnóstico del trastorno disocial de la conducta 
(CDTDC) creado por Pineda et al. (2000). En el presente trabajo hemos optado por denominarlo Cuestionario para el Diagnóstico del Trastorno de Conducta (CDTC) debido al cambio en la denominación observado en el DSM-5 (American Psychiatric Association, 2013). El mismo se compone de 14 reactivos que se responden con una escala tipo Likert de 4 posiciones y se califica de 0 (nunca) a 3 (siempre). Este instrumento es autoaplicado y breve. Fue creado en Medellín, donde se estudiaron sus propiedades psicométricas. Como se mencionó, se observó una estructura interna de tres factores, y mostró una adecuada consistencia interna $(\alpha=.86)$.

La Escala de Impulsividad (EI) de Plutchik, Van Praag, Picard, Conte y Korn (1989), que consta de 15 reactivos, los cuales hacen referencia a conductas como la sobrealimentación, la planeación, el control emocional, el control conductual, gastos impulsivos, entre otras. Sus 4 respuestas posibles puntúan en una escala Likert del 0 a 3 , y son nunca, algunas veces, frecuentemente y muy frecuentemente. Los puntajes del total oscilan entre $0 \mathrm{y}$ 45, donde valores elevados indican mayor impulsividad. En su versión mexicana (Páez et al., 1996) ha mostrado bajos valores de consistencia interna (valores de alfa de Cronbach entre .61 y .66) en población adulta.

La escala CRAFFT (por sus siglas en inglés; Car, Relax, Alone, Forget, Family/Friends, Trouble) de Knight, Sherrit, Shrier, Harris y Chang (2002) está diseñada para detectar problemas de abuso de alcohol o sustancias en adolescentes, respondiendo a preguntas que se dividen en dos partes. En la primera se obtiene información sobre el consumo de alcohol, marihuana y otras sustancias en el último año. Si el adolescente responde a una o más preguntas afirmativamente, debe responder a las siguientes 6 de la parte que se le presenta a continuación. El formato de respuesta es dicotómico (Si o No). Solo se puntúa la segunda parte; en caso de responder de forma negativa (no) se le asigna un puntaje de cero, mientras que a una respuesta afirmativa (sí) se le asigna un puntaje de uno. Si el individuo obtiene un total de 2 o más puntos, es decir dos o más respuestas afirmativas, indica que se necesita un seguimiento para la evaluación del problema. La escala ha sido adaptada en población mexicana (Reyna-Barajas, Copertari-Isaacson, González-Betanzos, \& Padrós-Blázquez, 2016) donde se confirmó una estructura unifactorial y mostró una consistencia interna satisfactoria $(\alpha=.73)$ y es usada a modo de cribaje.

El Inventario de depresión de Beck más conocido como BDI-Beck Depression Inventory-, es un instrumento con una larga tradición de uso y estudio (Beck, Steer, \& Carbin, 1988) que se compone de un total de 21 items (tipo Lickert, con una puntuación de 0 a 3), los cuales evalúan estado de ánimo, sentimientos de culpa, aislamiento social, preocupación somática, ideas suicidas, autoacusaciones, tendencia al llanto, indecisión, trastornos del sueño, del apetito y del deseo sexual entre otros. Fue empleado en su versión mexicana adaptada por Jurado et al., (1998), en la cual se obtuvo una consistencia interna de .87 (alfa de Cronbach) en la población general.

El State-Trait Anxiety Inventory (Inventario de ansiedad estado-rasgo) de Spielberger, Gorsuch y Lushene (1970) conocido en el ámbito hispanoparlante como Instrumento de Ansiedad Rasgo-Estado (IDARE) de Spielberger y DiazGuerrero (1975), es un cuestionario autoaplicado, diseñado para evaluar dos tipos de ansiedad (rasgo y estado). La escala de rasgo evalúa el nivel de ansiedad que habitualmente experimenta una persona (p. ej., "Cuando pienso en los asuntos que tengo entre manos me pongo tenso y alterado") y la de 
ansiedad estado mide la que se experimenta en el momento de la evaluación (p. ej., "Me siento ansioso" o "Me siento calmado", este último es un reactivo inverso). Consta de 40 items (20 items para evaluar el rasgo y los otros 20 el estado) con 4 posibles respuestas de tipo Likert que varían entre casi nunca y casi siempre para la escala de rasgo. Las opciones de respuesta para la escala de ansiedad-estado son: no, poco, regular y mucho (se obtienen de 0 a 3 puntos por reactivo). En ambas escalas, las puntuaciones altas indican presencia de elevados niveles de ansiedad. En la presente investigación se utilizó solo la que alude al estado. Las escalas han mostrado valores de alfa de Cronbach entre .75 y .88 para la ansiedad rasgo y de .82 a .90 para la de ansiedad estado en población adulta mexicana (Arias-Galicia, 1990).

\section{Procedimiento}

En la primera fase de esta investigación se revisó el CDTDC (la versión original colombiana) desarrollado por Pineda et al. (2000) por dos psicólogas nacidas y criadas en México. Se optó por modificar la redacción de algunos reactivos, se cambió el trato personal y se decidió usar un trato más directo (y menos formal) con la forma de "tú" en lugar del trato de "usted". Se consideró que esto era adecuado por si la formalidad en el trato pudiera influir en la comprensión de los reactivos o aumentar la defensividad en las respuestas de los sujetos. Además, en los reactivos 3, 5 y 12 se cambiaron palabras para una mejor comprensión: "vehículo" por "auto", "arremete" por "amenaza" y "atracado" por "amenazado" respectivamente.

El instrumento modificado, con las otras escalas (IE, BDI, CRAFFT e IDARE), se presentó junto con una hoja de oficio donde se explicaba la finalidad de la investigación y el carácter voluntario y anónimo de los participantes, con el objetivo de obtener autorización por parte de la dirección de una escuela de secundaria de la ciudad Morelia, Michoacán para aplicar los instrumentos a alumnos con edades entre 12 y 15, de ambos sexos de los tres grados. Los padres firmaron un consentimiento informado, donde permitian que sus hijos/as respondieran de forma voluntaria a los cuestionarios (en el que aceptaron no conocer los resultados, debido a que la evaluación era anónima). Se acudió a los distintos salones, y en un único encuentro se aplicaron los instrumentos: el CDTC, la Escala de Impulsividad de Plutchik, el Inventario de la escala CRAFFT, Depresión de Beck, y por último el Inventario de Ansiedad estado-rasgo (IDARE) con una duración total aproximada de 45 minutos. Los participantes de cada grupo respondieron de forma grupal, voluntaria y anónima.

Se generó la matriz de datos con la información recabada y se eliminaron aquellos cuestionarios que no fueron contestados en su totalidad (un total de 3). Por último, se realizaron los análisis estadísticos (Análisis de Componentes Principales, alfa de Cronbach, $r$ de Pearson y $t$ de Student Fisher) con el software Statistical Package for the Social Sciences (SPSS) versión 17.0 para Windows. 


\section{RESULTADOS}

\section{Estructura interna}

Con el objetivo de hallar la estructura interna a través de un análisis de componentes principales, se comprobó que los datos fueran adecuados para dicho análisis (Test de esfericidad de Bartlett $=1583.07$ con $p<.001$; indice Kaiser Meyer Olkin $=.903$ ) de acuerdo a los criterios de Kaiser.

Se estudió la distribución de los valores propios (eigenvalues) y el porcentaje de varianza explicada (ver tabla 1). El número de factores con valores propios superior a 1 fue de 3, lo que explica el $57.97 \%$ de la varianza. Se observó una gran diferencia entre el primer y el segundo factor, pero debido a que hasta tres factores obtuvieron un valor superior a la unidad, se revisaron las soluciones factoriales con 2 y 3 factores (haciendo uso de la rotación Varimax y Oblimin). Respecto a la versión de 2 factores se observó un elevado peso de 3 reactivos en ambos componentes. Al analizar la opción de 3 factores, se encontraron 4 items con una carga compartida en dos de los tres componentes. Además, acorde a los pesos factoriales obtenidos, era muy complicado interpretar y darle un significado a los factores resultantes (que no coincidieron con los hallados en la versión colombiana). Por ello y teniendo en cuenta el criterio de parsimonia, consideramos que la escala tiene un solo factor con una varianza explicada del $41 \%$. Puede observarse que las cargas factoriales de los reactivos son superiores a .50 (ver tabla 2).

Tabla 1.

Autovalores, varianza explicada y acumulada de los factores del CDTC.

\begin{tabular}{cccc}
\hline Componente & Autovalor & $\begin{array}{c}\text { \% Varianza } \\
\text { explicada }\end{array}$ & $\begin{array}{c}\text { \% Varianza } \\
\text { acumulado explicada }\end{array}$ \\
\hline 1 & 5.74 & 41.00 & 41.00 \\
2 & 1.26 & 9.03 & 50.04 \\
3 & 1.11 & 7.93 & 57.97 \\
4 & 0.82 & 5.90 & 63.87 \\
5 & 0.73 & 5.22 & 69.10 \\
\hline
\end{tabular}

Tabla 2.

Cargas factoriales de los 14 reactivos del CDTC.

\begin{tabular}{cc}
\hline Ítems & Factor único \\
\hline 1 & .51 \\
2 & .58 \\
3 & .66 \\
4 & .63 \\
5 & .68 \\
6 & .62 \\
7 & .54 \\
8 & .72 \\
9 & .63 \\
10 & .68 \\
11 & .61 \\
12 & .70 \\
13 & .64 \\
14 & .65 \\
\hline
\end{tabular}




\section{Consistencia interna e idoneidad de los ítems}

La escala mostró una adecuada consistencia interna, debido a que el valor de alfa de Cronbach resultó elevada $(\alpha=.87)$.

La puntuación media para cada ítem osciló entre los valores de 0.03 (item 12) y de 0.41 (item 6). Los valores de la desviación típica de cada reactivo oscilaron entre 0.33 y 0.62. El rango de correlación de cada uno de los ítems con la puntuación total corregida sin tener en cuenta dicho ítem, fueron todos superiores a .45; además, se observó que ningún ítem al ser eliminado hace aumentar el valor del alfa de Cronbach de la escala total. Por ello, puede concluirse que todos los reactivos son adecuados (ver tabla 3).

Tabla 3.

Media, desviación estándar, correlación del ítem con la escala total (corregida) y el valor del alfa de Cronbach si se elimina el ítem de los reactivos del CDTC.

\begin{tabular}{ccccc}
\hline Ítems & Media & $\begin{array}{c}\text { Desviación } \\
\text { típica }\end{array}$ & $\begin{array}{c}\text { Correlación } \\
\text { ittem escala } \\
\text { total (corregida) }\end{array}$ & $\begin{array}{c}\text { Alfa de la escala } \\
\text { total si se } \\
\text { elimina ítem }\end{array}$ \\
\hline 1 & 0.38 & 0.57 & .45 & .87 \\
2 & 0.10 & 0.37 & .49 & .86 \\
3 & 0.10 & 0.34 & .62 & .86 \\
4 & 0.17 & 0.46 & .56 & .86 \\
5 & 0.25 & 0.52 & .63 & .86 \\
6 & 0.41 & 0.61 & .57 & .86 \\
7 & 0.15 & 0.40 & .45 & .86 \\
8 & 0.10 & 0.36 & .64 & .86 \\
9 & 0.12 & 0.41 & .55 & .86 \\
10 & 0.17 & 0.46 & .61 & .86 \\
11 & 0.06 & 0.28 & .52 & .86 \\
12 & 0.03 & 0.21 & .58 & .86 \\
13 & 0.04 & 0.28 & .53 & .86 \\
14 & 0.10 & 0.35 & .56 & .86 \\
\hline
\end{tabular}

\section{Relación con otras variables y datos descriptivos}

Con la intención de obtener evidencias de validez de convergentediscriminante de la escala, se realizaron correlaciones de Pearson entre la escala CDTC y los otros instrumentos. Se hallaron valores moderados y positivos con la Escala de impulsividad de Plutchik $(r=.49)$, con el Inventario de depresión de Beck $(r=.48)$ y con el cuestionario que mide abuso de sustancias CRAFFT $(r=.47)$ —todas las correlaciones con $p<.001$ - Por otro lado, se observó ausencia de correlación con el Inventario de ansiedad estado $(r=-.09 ; p>.05)$.

Finalmente, se observó que la media del CDTC resultó de 2.16 ( $D E=$ 3.59), la moda fue de 0 , el valor mínimo fue de 0 y el máximo de 42 . Hasta un $21.6 \%$ de los evaluados obtuvieron una puntuación igual o superior a 4 . Véanse las frecuencias en la tabla 4.

Respecto a las posibles diferencias por género, se aplicó la $t$ de Student Fisher para datos independientes, donde se asumió que las varianzas eran iguales (debido a que se observó que en la prueba de Levene $F=2.47$; $p=$ .177), y los varones obtuvieron una puntuación $(M=2.58, D E=4.17)$ significativamente superior $(t=-1.99 ; p=.046)$ a la mostrada por los 
participantes del género femenino $(M=1.76, D E=2.89)$. Respecto a la edad se halló ausencia de correlación $(r=-.003 ; p=.960)$.

Tabla 4.

Frecuencias y porcentajes (simples y acumulados) de los valores de la puntuación total de la escala CDTC.

\begin{tabular}{ccccc}
\hline Valores & Frecuencia & $\%$ & $\begin{array}{c}\text { Frecuencia } \\
\text { acumulada }\end{array}$ & $\begin{array}{c}\text { Porcentaje } \\
\text { acumulado }\end{array}$ \\
\hline 0 & 108 & 35.4 & 108 & 35.4 \\
1 & 65 & 21.3 & 173 & 56.7 \\
2 & 44 & 14.4 & 217 & 71.1 \\
3 & 22 & 7.2 & 239 & 78.4 \\
4 & 25 & 8.2 & 264 & 86.6 \\
5 & 15 & 4.9 & 279 & 91.5 \\
6 & 8 & 2.6 & 287 & 94.1 \\
7 & 5 & 1.6 & 292 & 95.7 \\
8 & 5 & 1.6 & 297 & 97.4 \\
10 & 1 & 0.3 & 298 & 97.7 \\
12 & 1 & 0.3 & 299 & 98.0 \\
14 & 3 & 1.0 & 302 & 99.0 \\
17 & 1 & 0.3 & 303 & 99.3 \\
20 & 1 & 0.3 & 304 & 99.7 \\
42 & 1 & 0.3 & 305 & 100 \\
\hline
\end{tabular}

\section{DISCUSIÓN}

El objetivo principal del presente estudio fue adaptar y estudiar las características psicométricas del Cuestionario para el Diagnóstico del Trastorno de Conducta (CDTC) de Pineda et al. (2000) en una población general de adolescentes cuyas edades oscilan entre 12 y 15 años de Morelia, Michoacán (México), por la importancia que tiene el disponer de un instrumento con datos sobre su confiabilidad y evidencias de validez para la detección de casos de TC.

El instrumento se adaptó de la versión original colombiana de Pineda et al. (2000). Se modificó la redacción, cuando se consideró necesario, para mejorar la comprensión de la población mexicana. Esto se logró al utilizar sinónimos de algunas palabras en los reactivos 5 y 12 y también al cambiar el trato de "usted" al de "tú".

Respecto al estudio de las propiedades psicométricas, en primer lugar debe destacarse que la estructura interna del instrumento resultó unifactorial a diferencia de la versión colombiana, donde indican que hallaron 3 factores (violencia, violación de normas y crueldad). Sin embargo, haciendo uso de Análisis de Componentes Principales (con rotación Varimax y Oblimin), no se pudo hallar una solución satisfactoria con más de un factor. Nótese que al revisar la versión colombiana observamos que se introdujeron solo 10 de los 14 reactivos al realizar la rotación Varimax; así lograron una interpretación de tres factores. Mencionan que mantener los tres factores era lo adecuado ya que al realizar el mismo análisis con la mitad de la muestra se encontró la misma estructura. Pero debe destacarse que no contemplan 4 de los reactivos que forman parte de la escala y los mismos autores sugieren mantenerlos en el cuestionario. Creemos que quizás en un futuro podrian desarrollarse reactivos que tengan presente el callo emocional, que dispone de importante fundamentación empírica y teórica (Frick \& Nigg, 2012; Frick, Stickle, 
Dandreaux, Farrell, \& Kimonis, 2005; Frick \& White, 2008; Moffitt et al., 2008), y que encajarian en el especificador con emociones prosociales limitadas, que actualmente se contempla en el DSM-5 (American Psychiatric Association, 2013).

Respecto al objetivo 2 , referido a la consistencia interna y a la idoneidad de los reactivos, el alfa de Cronbach muestra una elevada y adecuada confiabilidad. El valor del índice hallado fue incluso ligeramente mayor al obtenido en el estudio de Pineda et al. (2000) lo cual puede deberse a que se simplificó la redacción de los ítems. Debe recordarse que todos los reactivos resultaron adecuados, ya que contribuian de forma favorable en el índice de confiabilidad total del cuestionario y todos manifestaron una correlación adecuada con el total de la escala.

Respecto a la relación con otras variables, se encontró una correlación moderada y positiva con la Escala de impulsividad de Plutchik, lo cual era esperable debido a que las conductas impulsivas son un criterio del TC según el DSM-5 (American Psychiatric Association, 2013) que se refiere a él como bajo autocontrol. Además, se ha señalado que ante la presencia de un problema de impulsividad se puede desarrollar un problema de conducta y de relaciones sociales, lo que conduce a deterioros significativos en las áreas académicas, familiares y sociales del individuo (Anney et al., 2008; Maciá, 2012), y se ha asociado frecuentemente al trastorno por déficit de atención e hiperactividad (Beauchaine et al., 2010; Ulloa et al., 2006).

Sobre la relación con la escala CRAFFT (Knight et al., 2002), también se observa una moderada correlación positiva entre el riesgo de abuso de alcohol y otras sustancias y el TC. Esto es congruente con lo que indica el DSM-5 (American Psychiatric Association, 2013): el abuso de sustancias es una característica, especialmente frecuente en las mujeres, con TC. Otra investigación que apoya la relación de abuso de sustancias con problemas de conducta es la de Torres de Galvis, Posada-Villa, Bareño-Silva y BerbesíFernández (2010), que señalan que el TC manifiesta una mayor relación con el trastorno por uso de sustancias.

Por otro lado, Flory y Lyam (2003) mencionan que existe un alto índice de abuso de sustancias en los adolescentes con TDAH, pero esta relación se reduce de forma significativa cuando se ha tratado de forma satisfactoria (controlado) la comorbilidad de un TC en el individuo. Frick (1998) indica que la asociación entre el abuso de sustancias y el TC es frecuente y de gran importancia. Otros estudios han reportado la relación entre el TC y el abuso de sustancias (Greenfield et al., 2017; Krueger, 1999; Ulloa et al., 2006; Van Kammen, Lober, \& Stouthamer-Loeber, 1991; Wiener \& Dulcan, 2006). Por lo que es evidente que existe una importante relación entre el TC y el abuso de sustancias, lo cual es congruente con la correlación hallada entre la escala CDTC y la escala CRAFFT.

En cuanto al Inventario de Depresión de Beck, también se halló una correlación positiva y moderada. Ello era también esperable porque como lo indica De la Peña-Olvera (2000), en individuos con sintomatología depresiva frecuentemente se observan problemas de conducta (como son la agresión y la violencia). Por otro lado, es notable la comorbilidad entre el TC y los trastornos depresivos (Abram et al., 2015; Avenevoli, Swendsen, He, Burstein, \& Merikangas, 2015; Krueger, 1999).

Respecto al inventario de Ansiedad estado no se halló correlación significativa con el CDTC. Russo y Biedel (1994) afirman que del 22 al $33 \%$ de 
los casos de niños con problemas de conducta manifiestan problemas de ansiedad y aquellos tratados clínicamente por el TC, entre un 65 y $75 \%$ también se les ha diagnosticado con el trastorno ansiedad. Debe señalarse que se reportó también una correlación baja entre el rasgo de ansiedad y características del TC (Fergusson \& Horwood, 1993; Krueger, 1999), lo cual no coincide con los resultados hallados en nuestra investigación. Por otro lado, en la investigación realizada por Frick, Lilienfeld, Ellis, Loney y Silverthorn (1999), que también reportaron una relación positiva y baja entre la ansiedad y los rasgos de $\mathrm{TC}$, hallaron una correlación negativa con el rasgo de callo emocional, lo que puede reducir e incluso anular la correlación si se controla la presencia de dicho rasgo. Quay (1987) menciona que aquellos niños que tienen ambas alteraciones (Trastornos de ansiedad y TC) han sido descritos como menos agresivos, presentan menor cantidad de ausencias escolares y tienen una mejor respuesta a los tratamientos, que aquellos que no presentan ansiedad. De esta manera, presentar ansiedad elevada podría ser un factor protector ante el $\mathrm{TC}$, debido a que la alta susceptibilidad al castigo (mayor temor) reduciría la probabilidad de realizar conductas antinormativas (con elevado riesgo de ser sancionadas). Los resultados hallados en esta investigación apoyan dicha perspectiva.

Por otro lado, la mayor presencia de sintomatología de TC observada en los varones respecto a las participantes femeninas coincide con los datos ofrecidos por la American Psychiatric Association (1994; 2013).

Es necesario señalar que en el presente estudio no se ha contado con un instrumento alterno válido y fiable que evalúe el TC directamente, ya que actualmente no existe ninguno en México. Sería deseable, especialmente en población clínica, hacer uso de un gold standard en un futuro, como el uso de una entrevista en profundidad realizada por un profesional experto que establezca un diagnóstico de TC en los participantes, lo que podría ser de gran utilidad para identificar un punto de corte que pudiera orientar el diagnóstico. Pero teniendo en cuenta las correlaciones mostradas por el CDTC con las diferentes variables, puede concluirse que muestra indicios favorables sobre la validez de convergente-discriminante de la escala. Sin embargo, se requiere más investigación para aseverar la validez del instrumento.

Se propone en futuras investigaciones estudiar la fiabilidad test-retest. También sería interesante ampliar la muestra incluyendo población con características sociodemográficas más variadas. Especialmente en población de riesgo, por ejemplo en adolescentes no escolarizados y adolescentes que viven y/o trabajan en la calle, así como en centros de internamiento.

Sería pertinente además estudiar la sensibilidad al cambio en aquellos adolescentes con TC que mejoraran de forma clinicamente significativa después de recibir un tratamiento, aplicando el instrumento antes y después del tratamiento.

Es importante mencionar que los instrumentos se aplicaron de manera anónima, lo cual favoreció la sinceridad ya que se redujo la defensividad del adolescente. En la valoración de las puntuaciones totales se observa que hasta un $21.6 \%$ de la muestra total obtiene una puntuación igual o superior a 4 , el punto de corte que sugieren los autores en la versión original (Pineda et al., 2000) de riesgo de TC. Dicho porcentaje es muy inferior al observado en la muestra original (como se mencionó anteriormente, se estimó, resultando superior al $45 \%$ ); ello puede deberse a que en la versión original se estratificó por clase social y toda la muestra estuvo compuesta exclusivamente por 
varones. También debe mencionarse que la administración del cuestionario fue anónima, es posible que los resultados cambien si el evaluado se identifica (debido a la presencia de mayor defensividad). Sería interesante estudiar las diferencias en las puntuaciones en adolescentes identificados respecto a aquellos que responden de forma anónima. Es de interés sugerir que la aplicación anónima de este instrumento puede ser de gran utilidad para detectar riesgos en instituciones e incluso para estudios de tipo sociológico.

Para concluir, deseamos destacar que a partir de los resultados de la presente investigación se puede concebir a la escala CDTC, en su versión preliminar, como una herramienta con aceptables propiedades para el cribaje del TC.

\section{REFERENCIAS}

Abram, K. M., Zwecker, N. A., Welty, L. J., Hershfield, J. A., Dulcan, M. K., \& Teplin, L. A. (2015). Comorbidity and continuity of psychiatric disorders in youth after detention: a prospective longitudinal study. JAMA psychiatry, 72(1), 84-93. https://doi.org/10.1001/jamapsychiatry.2014.1375

American Psychiatric Association (1994). Diagnostic and statistical manual of mental disorders (4th ed.). Washington DC: Autor.

American Psychiatric Association (2000). Diagnostic and statistical manual of mental disorders (4th. ed., text revision). Washington, DC: Autor.

American Psychiatric Association (2013). Diagnostic and statistical manual of mental disorders (5th ed.). Washington, DC: Autor. https://doi.org/10.1176/appi.books.9780890425596

Anney, R. J., Lasky-Su, J., O'Dúshláine, C., Kenny, E., Neale, B. M., Mulligan, A.,... Gill, M. (2008). Conduct disorder and ADHD: evaluation of conduct problems as a categorical and quantitative trait in the international multicentre ADHD genetics study. American journal of medical genetics, 147B(8), 13691378. https://doi.org/10.1002/ajmg.b.30871

Arias-Galicia, F. (1990). Investigaciones sobre el IDARE en cuatro países latinoamericanos: Argentina, Ecuador, México y Perú. Revista Intercontinental de Psicología y Educación, 3, 49-85.

Avenevoli, S., Swendsen, J., He, J. P., Burstein, M., \& Merikangas, K. R. (2015). Major depression in the National Comorbidity Survey-Adolescent Supplement: prevalence, correlates, and treatment. Journal of the American Academy of Child \& Adolescent Psychiatry, 54(1), 37-44. https://doi.org/10.1016/j.jaac.2014.10.010

Auerbach, R. P., Alonso, J., Axinn, W. G., Cuijpers, P., Ebert, D. D., Green, J. G.,... Bruffaerts, R. (2016). Mental disorders among college students in the World Health Organization World Mental Health Surveys. Psychological medicine, 46(14), 1-16. https://doi.org/10.1017/S0033291716001665

Beauchaine, T. P., Hinshaw, S. P., \& Pang, K. L. (2010). Comorbidity of attention-deficit/hyperactivity disorder and early-onset conduct disorder: biological, environmental, and developmental mechanisms. Clinical Psychology: Science \& Practice, 17, 327-336. https://doi.org/10.1111/j.14682850.2010.01224.x

Beck, A. T., Steer, R. A., \& Carbin, M. G. (1988). Psychometric properties of the Beck Depression Inventory: Twenty-five years of evaluation. Clinical psychology review, 8(1), 77-100. https://doi.org/10.1016/0272-7358(88)90050-5

De la Peña-Olvera, F. R. (2000). El trastorno por déficit de atención con hiperactividad (TDAH). Revista de la Facultad de Medicina UNAM, 43, 243-244.

Eyberg, S. (1992). Parent and teacher behavior inventories for the assessment of conduct problem behaviors in children. Innovations in clinical practice: A source book, 11, 261-270.

Fergusson, D. M. \& Horwood, L. J. (1993). The structure, stability and correlations of the trait components of conduct disorder, attention deficit and anxiety/withdrawal reports. Journal of Child Psychology and Psychiatry, 34(5), 749-766. https://doi.org/10.1111/j.1469-7610.1993.tb01069.x

Flory, K. \& Lynam, D. R. (2003). The Relation Between Attention Deficit Hyperactivity Disorder and Substance Abuse: What Role Does Conduct Disorder Play? Clinical Child and Family Psychology Review, 6, 1-16. https://doi.org/10.1023/A:1022260221570

Frick, P. J. (1998). Conduct Disorders and Severe Antisocial Behavior. New York: Plenum Press. https://doi.org/10.1007/978-1-4615-5343-4

Frick, P. J., Lilienfeld, S. O., Ellis, M., Loney, B., \& Silverthorn, P. (1999). The association between anxiety and psychopathy dimensions in children. Journal of abnormal child psychology, 27(5), 383-392. https://doi.org/10.1023/A:1021928018403

Frick, P. J. \& Nigg, J. T. (2012). Current issues in the diagnosis of attention deficit hyperactivity disorder, oppositional defiant disorder, and conduct disorder. Annual Review of Clinical Psychology, 8, 77-107. https://doi.org/10.1146/annurev-clinpsy-032511-143150

Frick, P. J., Stickle, T. R., Dandreaux, D. M., Farrell, J. M., \& Kimonis, E. R. (2005). Callous-unemotional traits in predicting the severity and stability of conduct problems and delinquency. Journal of Abnormal Child Psychology, 33, 471-487. https://doi.org/10.1007/s10648-005-5728-9 
Frick, P. J. \& White, S. F. (2008). Research review: The importance of callous-unemotional traits for the development of aggressive and antisocial behavior. Journal of Child Psychology and Psychiatry, 49, 359-375. https://doi.org/10.1111/j.1469-7610.2007.01862.x

Greenfield, B. L., Sittner, K. J., Forbes, M. K., Walls, M. L., \& Whitbeck, L. B. (2017). Conduct disorder and alcohol use disorder trajectories, predictors, and outcomes for indigenous youth. Journal of the American Academy of Child \& Adolescent Psychiatry, 56(2), 133-139. https://doi.org/10.1016/j.jaac.2016.11.009

Jurado, S., Villegas, M.E., Méndez, L. Rodríguez, F. Loperena, V., \& Varela, R. (1998). La estandarización del Inventario de Depresión de Beck para los residentes de la ciudad de México. Salud Mental. 21(3), 26-31.

Knight, J. R., Sherritt, L., Shrier, L. A., Harris, S. K., \& Chang, G. (2002). Validity of the CRAFFT substance abuse screening test among adolescent clinic patients. Archives of pediatrics \& adolescent medicine, 156(6), 607-614. https://doi.org/10.1001/archpedi.156.6.607

Krueger, R. F. (1999). The structure of common mental disorders. Archives of General Psychiatry, 56(10), 921-926. https://doi.org/10.1001/archpsyc.56.10.921

Maciá, D. (2012). TDAH en la infancia y la adolescencia. Concepto, evaluación y tratamiento. Madrid: Ediciones Pirámide.

McNeil, C. B. \& Hembree-Kigin, T. L. (2010). Parent-Child Interaction Therapy. New York: Springer. https://doi.org/10.1007/978-0-387-88639-8

Medina-Mora, M.E., Borges, G., Lara-Muñoz, C., Benjet, C., Blanco-Jaimes, J., Fleiz-Bautista, C.,... AguilarGaxiola, S. (2003). Prevalencia de trastornos mentales y uso de servicios: Resultados de la Encuesta Nacional de Epidemiologia Psiquiátrica en México. Salud Mental, 26(4), 1-16.

Moffitt, T.E., Arseneault, L., Jaffee, S.R., Kim-Cohen, J., Koenen, K.C., Odgers, C.L.,... Viding, E. (2008). Research review: DSM-V conduct disorder: research needs for an evidence base. Journal of Child Psychology and Psychiatry, 49, 3-33. https://doi.org/10.1111/j.1469-7610.2007.01823.x

Páez, F., Jiménez, A., López, A., Ariza, J. P., Soto, H. O., \& Nicolini, H. (1996). Estudio de validez de la traducción al castellano de la Escala de Impulsividad de Plutchik. Salud Mental, 19(3), 10-12.

Padrós-Blázquez, F., Rosiles-Leyva, F. P., Hernández-Luján, E., Herrera-Guzmán, I., Martínez-Medina, M. P., Gudayol-Ferré, E., \& Villuendas González, E. R. (2015). Trastorno disocial: un origen de la agresividad patológica. En M. E. Murueta \& M. Orozco-Guzmán (Eds.), Psicología de la violencia; Causas, Prevención y afrontamiento (tomo I) (pp. 77-92). México: Manual Moderno.

Padrós Blázquez, F., Zamora Cervantes, M., Salinas García, P., \& Martinez Medina, M. P. (2017). Cinco vías del TDAH al consumo patológico de alcohol. Cuadernos de medicina psicosomática y psiquiatría de enlace, 121, 27-35.

Pineda D. A., Puerta I. C , Arango C. P., Calad O. M., \& Villa M. T. (2000). Un cuestionario breve para el diagnóstico del trastorno disocial de la conducta en adolescentes de 12 a 16 años. Revista de Neurología, 30, 1145-1150.

Posada-Villa, J. A., Aguilar-Gaxiola, S. A., Magaña, C. G., \& Gómez, L. C. (2004). Prevalencia de trastornos mentales y uso de servicios: resultados preliminares del Estudio nacional de salud mental Colombia 2003. Revista Colombiana de Psiquiatría, 33, 241-262.

Plutchik, R., Van Praag, H. M., Picard, S., Conte, H. R., \& Korn, M. (1989). Is there a relation between the seriousness of suicidal intent and the lethality of the suicide attempt? Psychiatry research, 27(1), 7179. https://doi.org/10.1016/0165-1781(89)90011-5

Quay, H. (1987). Handbook of Juvenile Delinquency. New York: Wiley.

Redondo, S., Garrido, V., Pérez, J., \& Barberet, R. (1997). Advances in Psychology and Law: International Contributions. Berlin: Walter de Gruyter. https://doi.org/10.1515/9783110801163

Redondo, S. \& Sánchez-Meca, J. (2003). Guía de tratamientos psicológicos eficaces para la delincuencia juvenil. En M. Pérez Alvarez, J. R. Fernández Hermida, C. Fernández Rodríguez \& I. Amigo (Eds.), Guía de tratamientos psicológicos eficaces III. Infancia y adolescencia (pp. 183 -214). Madrid: Pirámide.

Reyna-Barajas, G. V., Copertari-Isaacson, L.F., González-Betanzos, F., \& Padrós-Blázquez, F. (2016). Estudio psicométrico del instrumento CARLOS (CRAFFT) en estudiantes universitarios mexicanos. Evaluar, 16, 10-19.

Russo, M. F. \& Beidel, D. C. (1994). Comorbidity of childhood anxiety and externalizing disorders: Prevalence, associated characteristics, and validation issues. Clinical Psychology Review, 14, 199-221. https://doi.org/10.1016/0272-7358(94)90008-6

Spielberger, C. D. \& Díaz-Guerrero, R. I. (1975). Inventario de ansiedad: rasgo-estado. México: El Manual Moderno.

Spielberger, C.D., Gorsuch, R.L., \& Lushene, R. E. (1970). Manual for the state-trait anxiety inventory. Palo Alto, CA: Consulting Psychologists Press.

Staff, V. V., Machleidt, W., \& Bauer, M. (2004). Psiquiatría, trastornos psicosomáticos y psicoterapia. España: Elsevier.

Thornberry, T. P. \& Krohn, M. D. (2000). The Self-Report Method for Measuring Delinquency and Crime. Criminal Justice, 4(1), 33-83.

Torres de Galvis, Y., Posada-Villa, J., Bareño-Silva, J., \& Berbesí-Fernández, D. (2010). Trastornos por abuso y dependencia de sustancias en población colombiana: su prevalencia y comorbilidad con otros trastornos mentales seleccionados. Revista Colombiana de Psiquiatria, 39, 14-35. https://doi.org/10.1016/S0034-7450(14)60265-1

Ulloa, R. E., Sánchez, S., Sauceda, J. M., \& Ortiz, S. (2006). Psicopatología asociada al trastorno por déficit de atención e hiperactividad en niños de edad escolar. Actas Españolas de Psiquiatria, 34, 330-335.

Van Kammen, W., Lober, R., \& Stouthamer-Loeber, M. (1991). Substance Use and Its Relationship to Conduct Problems and Delinquency in Young Boys. Journal of Youth and Adolescence, 20, 399-413. 
Padrós-Blázquez, F., Olavarrieta, A. D., Martinez-Medina, M. P. \& González-Betanzos, F. / Psicodebate, 18(1), $7-20$.

https://doi.org/10.1007/BF01537182

Wiener, J. M. \& Dulcan, M. K. (2006). Tratado de psiquiatria de la infancia y la adolescencia. Barcelona: Masson.

Recibido 26-06-2017 | Aceptado 31-10-2017 